\title{
Coronal transients and metric type II radio bursts
}

\section{Accelerations at low coronal heights}

\begin{abstract}
S. Mancuso
INAF, Osservatorio Astronomico di Torino, Strada Osservatorio 20, 10025 Pino Torinese, Italy e-mail: mancuso@oato.inaf.it

Received 23 December 2005 / Accepted 24 October 2006

ABSTRACT

In the companion Paper I, Mancuso \& Raymond (2004) investigated the relationship between type II and coronal mass ejection (CME) activity for a sample of twenty-nine CME/shock events that occurred between March and December 1999. Most of the events appeared to lead the type II emission locations by several minutes and the two sets of speeds were not well-correlated, in apparent disagreement with a CME-driven origin interpretation of the coronal shocks. The above discrepancies were attributed to an artifact effect due to geometry, favoring emission at the flanks of the CME leading edges in correspondence with denser low-Alfvén-speed coronal structures, where shock strengths are enhanced. An important caveat in the analysis carried out in Paper I is that the conclusions that supported the CME-driven shock front-flank scenario were based on sunward-extrapolated CME trajectories deduced from the analysis of Large Angle and Spectrometric Coronagraph (SOHO/LASCO) observations in the outer corona that might not be accurate at low coronal heights where significant accelerations should be present before the CMEs acquire the speeds inferred by coronagraphic images. In the present paper, we re-examine the above relationship for a subset of ten events by integrating the LASCO measurements with ancillary CME observations taken by other instruments (MLSO/Mk4, SOHO/EIT, and UVCS) at coronal heights comparable to the typical type II radio emissions (1.2-2.5 $R_{\odot}$ ). We investigate the bias introduced in the sunward extrapolation of LASCO data and discuss whether the presence of previously undetected CME accelerations at low coronal heights might have affected the conclusions put forth in the CME-driven shock front-flank scenario proposed in Paper I. We show that the new set of observations neither solve the problem of the timing between CMEs and shocks nor improve the correlation between CME and shock speeds, although acceleration effects are found to be important at the typical metric type II heights and must be taken into account for a proper analysis of the $\mathrm{CME} /$ shock relationship.
\end{abstract}

Key words. Sun: corona - Sun: radio radiation - Sun: coronal mass ejections (CMEs) - shock waves

\section{Introduction}

It is now well-established that kilometric type II radio bursts are manifestations of interplanetary shock waves formed in front of coronal mass ejections (CMEs) through a piston-driven mechanism (e.g., Sheeley et al. 1985). However, the formation process of coronal shocks, which produce type II emission in the metric wavelength range, is not yet fully understood and remains somewhat controversial. Direct positional comparisons between metric type II sources and coronal transients have often shown that the radio emission appears to be produced well behind CME leading edges. These observations, together with the evidence of a temporal association with solar flares, have induced several authors to speculate that metric type II radiation is produced by blast waves traveling through the ejected material and initiated during the impulsive phase of the associated flares (Uchida 1974; Wagner \& MacQueen 1983).

In a previous paper, Mancuso \& Raymond (2004; hereafter Paper I) investigated the relationship between type II and coronal transient activity in terms of emission originating from the top or the flanks of a shock surface, extending just above the coronal mass ejection (CME) leading edge surface. For this purpose, they analyzed ground-based metric type II radio bursts from twenty-nine CME events that occurred between March and December 1999 in conjunction with Large Angle and Spectrometric Coronagraph
(SOHO/LASCO; Brueckner et al. 1995) and UltraViolet Coronagraph Spectrometer (SOHO/UVCS; Kohl et al. 1995) observations. While LASCO C2/C3 observations were used to establish the correct geometry and dynamics of the CMEs, UVCS observations supplied accurate diagnostics of the pre-shocked ambient coronal electron density in the regions of propagation of the associated type II bursts (e.g., Mancuso et al. 2002, 2003), thus allowing a more reliable estimate of both heights and speeds of the CME-associated shocks with respect to previous studies that used general models for the ambient coronal density profiles. Mancuso \& Raymond (2004) used linear (constant speed) and quadratic (constant acceleration) sunward extrapolations of the weighted least-square fits to the height-time CME trajectories to compare the $\mathrm{CME}$ and shock dynamics, showing that most of the white-light leading edges appeared to lead the associated type II radio emission locations by several minutes (as predicted in the blast wave scenario) and that CME and type II speeds were not well-correlated, as instead it would be expected for CME-driven shocks.

In Paper I, the apparent lack of relationship that was found between metric type II bursts and CMEs was attributed to geometrical effects, favoring emission at the flanks of the shock surfaces, in correspondence with denser coronal structures. In fact, shock strengths can be very enhanced along streamer axes because of their lower Alfvén speeds (e.g., Kahler \& Reames 2003), and these streamers are typically offset from the axes 
Table 1. Parameters of the CME/flare/type II burst events considered in this work. See the text for further explanation.

\begin{tabular}{cc|cc|cccc}
\hline \hline \multicolumn{2}{c|}{ Type II burst } & \multicolumn{3}{|c|}{ Flare } & \multicolumn{3}{c}{ Coronal Mass Ejection } \\
\hline Date & $\begin{array}{c}\text { Start End } \\
\text { (UT) }\end{array}$ & $\begin{array}{c}\text { Onset Peak } \\
(\mathrm{UT})\end{array}$ & Location & $\begin{array}{c}\text { SOHO/LASCO } \\
(\mathrm{UT})\left(R_{\odot}\right)\end{array}$ & $\begin{array}{c}\text { SOHO/UVCS } \\
(\mathrm{UT})\left(R_{\odot}\right)\end{array}$ & $\begin{array}{c}\text { SOHO/EIT } \\
(\mathrm{UT})\left(R_{\odot}\right)\end{array}$ & $\begin{array}{c}\text { MLSO/Mk4 } \\
(\mathrm{UT})\left(R_{\odot}\right)\end{array}$ \\
\hline Mar. 08 & $06: 3806: 51$ & $06: 3006: 37$ & S24 E93 & $06: 54(2.30)$ & $\ldots$ & $06: 36(1.29)$ & $\ldots$ \\
Jun. 11 & $11: 1511: 31$ & $11: 0711: 57$ & E limb & $11: 26(2.74)$ & $\ldots$ & $11: 12(1.28)$ & $\ldots$ \\
Jul. 19 & $08: 4008: 44$ & $08: 1608: 46$ & N18 E59 & $09: 06(3.51)$ & $08: 40(1.86)$ & $\ldots$ & $\ldots$ \\
Aug. 04 & $05: 5105: 58$ & $05: 4505: 57$ & S16 W64 & $06: 26(2.31)$ & $\ldots$ & $06: 00(1.30)$ & $\ldots$ \\
Aug. 06 & $16: 4016: 46$ & $16: 2816: 36$ & S30 W85 & $18: 42(10.3)$ & $16: 41(2.47)$ & $\ldots$ & $\ldots$ \\
Aug. 20 & $18: 3918: 47$ & $18: 2518: 29$ & S23 E66 & $18: 50(3.29)$ & $\ldots$ & $\ldots$ & $18: 41(2.33)$ \\
Sep. 21 & $03: 1203: 15$ & $03: 0003: 32$ & N19 W90 & $03: 30(4.48)$ & $\ldots$ & $03: 12(1.29)$ & $\ldots$ \\
Oct. 23 & $01: 2501: 42$ & $00: 4801: 07$ & W limb & $01: 27(3.06)$ & $01: 14(1.90)$ & $\ldots$ & $\ldots$ \\
Oct. 26 & $21: 3021: 50$ & $21: 0921: 25$ & W limb & $21: 50(2.68)$ & $\ldots$ & $\ldots$ & $21: 21(1.66)$ \\
Oct. 27 & $13: 2513: 38$ & $13: 2413: 37$ & E limb & $13: 50(2.53)$ & $\ldots$ & $13: 25(1.38)$ & $\ldots$ \\
\hline
\end{tabular}

of symmetry along which the CMEs propagate. By applying a semiempirical model that takes the observed geometry of the CME expansion into account, they showed that the time lags between the type II and the leading edge passages in the lower corona could be consistently minimized and that the correlation between type II and CME speeds (or better, the speeds of those portions of the leading edges that intersect the streamer axes) was considerably improved.

Unfortunately, metric radio observations and white-light observations generally do not overlap. In the available LASCO C2 observations, CME leading edges are first detected well above the typical metric type II heights due to the limit set by the presence of the occulter that blocks the coronal emission below about $2 R_{\odot}$. As already pointed out in Paper I, the use of linear and quadratic sunward extrapolations of the CME trajectories is probably inaccurate near the surface of the Sun since significant accelerations must be present before the CMEs acquire the speeds inferred by coronagraphic images (St. Cyr et al. 1999; Zhang et al. 2001; Neupert et al. 2001; Gallagher et al. 2003; Shanmugaraju et al. 2003). Specifically, if the CMEs accelerated below a certain coronal height limited by the LASCO C2 occulter, the time lags between the type II emissions and the associated leading edges, as calculated in Paper I, could have been heavily overestimated, thus affecting the results of Mancuso \& Raymond (2004).

Luckily enough, ten out of the twenty-nine CME events analyzed by Mancuso \& Raymond (2004) were also observed in the inner corona by other instruments. In Sect. 2, we describe the new set of CME observations at the typical metric type II heights from the SOHO/UVCS, SOHO/EIT, and MLSO/Mk4 instruments. In Sect. 3, with the help of these observations, we reanalyze ten out of the twenty-nine events of the original sample in order to investigate the importance of acceleration effects below the LASCO C2 occulter and their influence on the results found in Paper I. Finally, in Sect. 4 we draw our conclusions.

\section{CME observations in the inner corona}

A thorough description of the set of observations used for this study and details about the analysis of the radio, ultraviolet, and white-light data related to all the CME/shock events of the sample can be found in Paper I and will not be repeated here. Table 1 lists the properties of the subset of ten $\mathrm{CME} /$ shock events analyzed in this work. The association of type II burst data with solar flares and CMEs was inferred according to closeness in time. The first three columns give information on the type II burst date and the start and end UT times. The next three columns provide the flare information (onset, peak, and location) obtained from the listings of the National Geophysical Data Center (NGDC). Finally, the last four columns give information on the time and height (in $R_{\odot}$ ) of first appearance of the CME in the fields of view of LASCO, UVCS, EIT, and Mk4.

\subsection{MLSO/Mk4 observations}

Two of the CMEs examined in Paper I (August 20 and October 26) were also detected in the inner corona by the Mark-IV (Mk4) white-light K coronameter at the Mauna Loa Solar Observatory (MLSO; Elmore et al. 2003). MLSO data are available to the community via website at the address http: //mlso. hao. ucar. edu. Figure 1a shows an Mk4 difference image of the leading edge of the October 26 event observed within the field of view of the instrument (from 1.12 to $2.79 R_{\odot}$ ) at 21:30 UT on the West limb. The CME leading edge, according to the list of activity seen by the observers at the MLSO, was visible in several exposures between 21:15 and 22:25 UT, expanding with an approximate width of about 34 degrees at $1.9 R_{\odot}$. Figure $1 \mathrm{~b}$ is a composite EIT and LASCO C2 difference image showing the evolution of the same CME about an hour later. As for the event on August 20, according to the MLSO observers, the Mk4 coronameter was able to detect CME material from 18:33 to 19:24 UT, expanding with an approximate width of about 40 degrees on the East limb. In this case, unlike the October 26 event, the full leading edge was hardly distinguishable in the difference images against the white-light background, so we were able to infer its height in just a couple of exposures taken at 18:41 and 18:48 UT.

\subsection{SOHO/UVCS observations}

Three of the CMEs examined in Paper I (July 19, August 6, and October 23) were also detected by UVCS. From an analysis of the line intensity variations of the $\mathrm{O}$ VI and $\mathrm{H}$ I Lyman $\alpha$ spectral lines at the CME/shock passage (e.g., Mancuso et al. 2002), we were able to infer the time at which each leading edge crossed the UVCS slit (see Table 1 for details). The July 19, August 6, and October 23 events were observed well below the heights corresponding to the first appearances of the CMEs in the field of view of LASCO C2, at approximately the heights of the associated type II sources, thus allowing a better comparison of the CME trajectory at the shock formation heights. In Fig. 2a, superimposed on a composite EIT and LASCO C 2 difference image of the July $19 \mathrm{CME} /$ shock event at 9:06 UT, we show the position of the UVCS slit on the East limb at the time of the observation. Figure $2 b$ is a composite EIT and LASCO C2 difference image showing a later phase of the evolution of the same CME. 

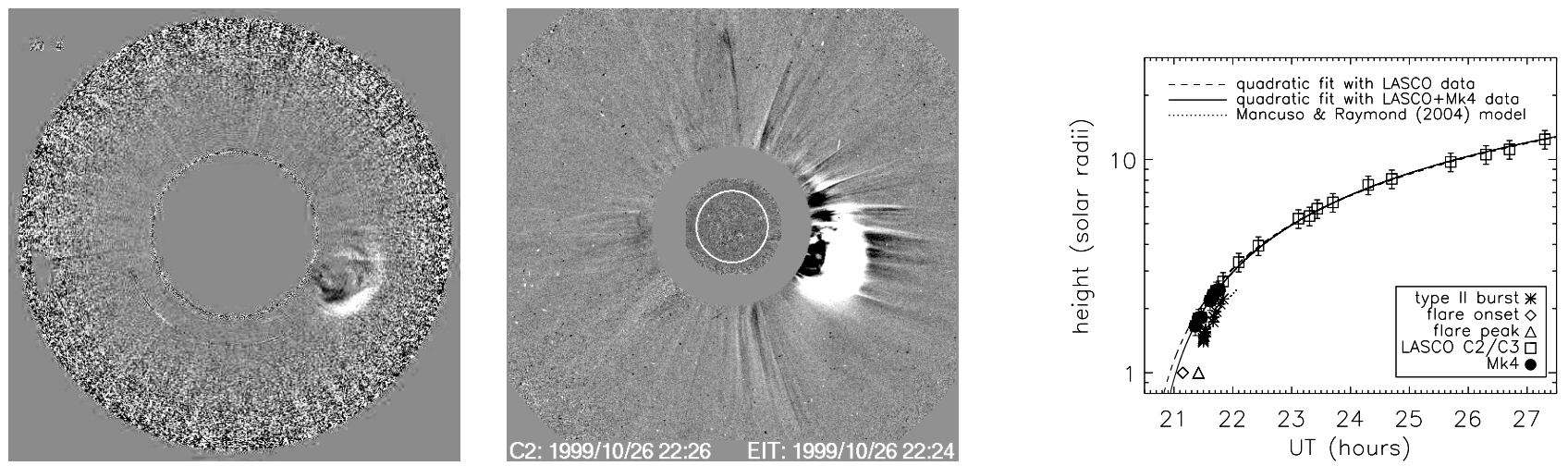

Fig. 1. a) Mk4 difference image showing the 1999 October $26 \mathrm{CME}$ at 21:30 UT in white-light. b) Composite EIT and LASCO C2 difference image showing the same CME at 22:26 UT. c) Height-time plot of the CME leading edge derived from LASCO observations (square symbols) and Mk4 observations (filled circles). The solid line is a least-square, second-degree polynomial fit to all (LASCO + Mk4) data; the dashed line is a second-degree polynomial fit to only LASCO data. The type II inferred heights (stars) are also plotted. The model trajectory of the type II burst (see Paper I) is represented by dotted lines. The onset time (diamond) and maximum time (triangle) of the associated flares are also indicated.
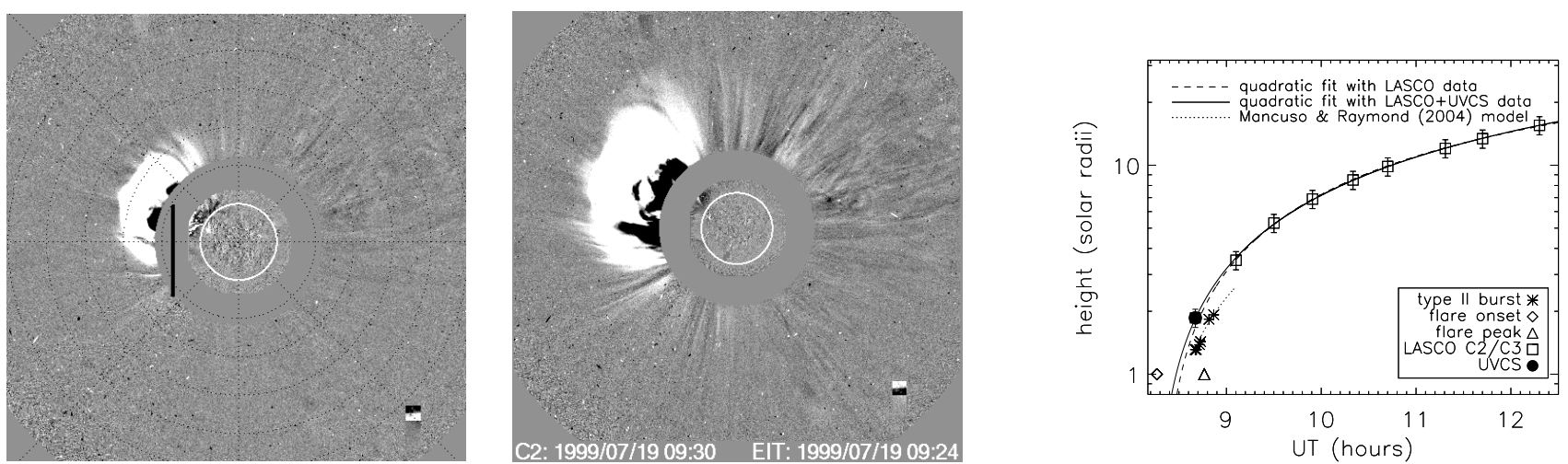

Fig. 2. a) UVCS slit position during the observation of the 1999 July 19 CME plotted over a composite EIT and LASCO C2 difference image showing the same CME at 9:06 UT. b) Composite EIT and LASCO C2 difference image showing a later phase of the evolution of the CME. c) Same as Fig. 1c.
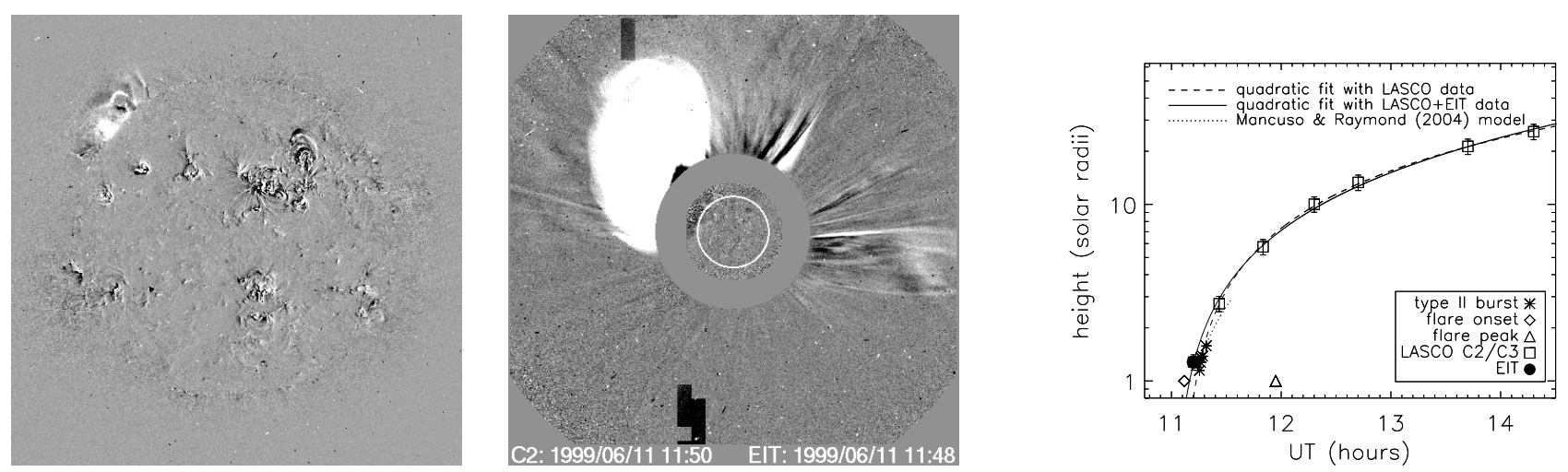

Fig. 3. a) EIT $195 \AA ̊$ difference image showing the eruption of the 1999 June 11 CME at 11:12 UT. b) Composite EIT and LASCO C2 difference image showing the structure of the CME at a later time. c) Same as Fig. 1c.

\subsection{SOHO/EIT observations}

Five of the CME events (March 8, June 11, August 4, September 21, and October 27) had features detected by the Extreme-ultraviolet Imaging Telescope (EIT) on SOHO (Delaboudinière et al. 1995) taken in the Fe XII $195 \AA$ line, related to ejected CME material. It is possible that the plasma and prominence material probed by EIT was lagging somewhat behind the leading edge detected by LASCO. In this sense, the CME heights estimated by EIT should probably be considered as lower limits to the actual heights of the leading edges. Figure $3 \mathrm{a}$ shows an EIT difference image of the eruption of CME material related to the June 11 event. Figure $3 b$ is a composite EIT and LASCO C2 difference image showing the structure of the CME at a later time.

\section{Results and discussion}

The new set of observations in the inner corona provided by the UVCS, EIT, and Mk4 instruments yields a better view of the 
earlier phases of the CME dynamics, allowing a probe of the acceleration effects below the LASCO C2 occulter when studying the $\mathrm{CME} / \mathrm{shock}$ relationship. This new information enabled us to test the limits in adopting quadratic extrapolations to the height-time CME trajectories at the typical metric type II heights and to investigate whether previously unaccounted-for accelerations at low coronal heights below the LASCO C2 coronagraph field of view might have critically affected the conclusions put forth in the CME-driven shock front-flank scenario proposed by Mancuso \& Raymond (2004), where the type II emission altitudes were directly compared to the sunward extrapolated CME trajectories.

Figure 1c shows the height-time trajectory of the leading edge derived from LASCO observations (square symbols) and Mk4 observations (filled circles) for the October 26 event. As in Paper I, the CME trajectories in the outer corona were inferred from LASCO C2/C3 height-time measurements available from the online LASCO CME catalog (Yashiro et al. 2002). In this case, the CME heights estimated from the analysis of the Mk4 data are consistent with those of LASCO. The dashed line is a least-square, second-degree polynomial fit to the LASCO data, while the solid line is a similar fit to the combined LASCO and Mk4 observations. Type II radio burst heights were deduced by using density estimates derived from the UVCS diagnostics, as in Paper I. The type II inferred heights are plotted, together with the model trajectory of the shock obtained by finding the angle correction that minimizes the time lag between the type II and the associated $\mathrm{CME}$ at $1.8 R_{\odot}$ and by assuming a shock surface wrapped around the leading edge. The onset time and maximum time of the associated flare are also indicated for completeness. Figures $2 \mathrm{c}$ and $3 \mathrm{c}$ show similar height-time trajectories of the leading edges for the July 19 and June 11 events, but with complementary UVCS and EIT observations, respectively. Figures $1-3 \mathrm{c}$ demonstrate that the adopted quadratic extrapolations (at least for these three events) was a fair (though not exhaustive) proxy of the CME trajectories even at the typical shock formation heights.

We note that correct estimates of density profiles are essential for a proper comparison of the CME and shock dynamics since the plasma frequency, indicative of the type II heliocentric height, is proportional to the square root of the density of the background, pre-shocked coronal plasma, which can vary as much as an order of magnitude during the rising phase to solar maximum (Mancuso et al. 2003). Derivation of the shock speeds thus requires previous knowledge of the density profile of the coronal structures through which the shocks propagate. The shock dynamics implied by the metric type II radio emission is determined through the drift rate at the frequency of observation and the electron density gradient in the corona at the plasma level corresponding to the observing frequency. The electron density is usually taken from a model, e.g., Newkirk (1961) or Saito (1970) models multiplied by an arbitrary factor. Consequently, the derivation of the shock speed depends closely on the assumed density model, and the associated uncertainty could be so high that both the estimated shock speeds and the time delays between the type II and the CME passages might be strongly affected.

In order to overcome this problem, as in Paper I, we used the refined density diagnostics offered by the SOHO/UVCS observations of the $\mathrm{O}$ VI doublet line intensities to infer the density profiles of the pre-shocked plasma structures just before the passage of each shock (e.g., Mancuso et al. 2003). By taking into account uncertainties in temperature and statistical errors in spectral line intensities, the coronal streamer electron densities are determined to within $20 \%$ (i.e., Bemporad et al. 2003). The corresponding uncertainties in the type II heights are thus on the order of $0.06 R_{\odot}$ at $50 \mathrm{MHz}$, which is a typical frequency of a metric type II burst (fundamental lane). It must be acknowledged that radio spectrograph measurements do not yield direct knowledge of the spatial position of the type II exciters, so that the quoted electron density uncertainties may be too optimistic. In fact, densities along the streamer axes are typically higher than at the edges by a factor of 1.5-2 (Bemporad et al. 2003). However, considering both that shock strengths (and consequently type II emissions) are expected to be enhanced along streamer axes because of their lower Alfvén speeds and that our density estimates refer to these portions of the streamers, the quoted uncertainties should be accurate enough. The speeds (and relative errors) of the type II bursts were thus inferred by fitting a weighted straight line (assuming constant speed, as usual) to the type II heights as derived from the analysis of the available radio dynamic spectra. In this way, the bias related to the unknown density profile was completely removed and, consequently, the resulting uncertainties in the results discussed in this paper are not dependent on an assumed density model. It is also worth emphasizing that, although the density diagnostics provided by UVCS is an average along the line of sight, it is strongly dominated by the streamers near the plane of the sky. For this reason, we only selected limb events, thus eliminating any possible bias related to line-ofsight effects. This is an advantage compared to white-light (e.g., $\mathrm{SOHO} / \mathrm{LASCO}$ ) density estimates for which it is necessary to assume cylindrical symmetry in order to extract the streamer's electron density profile.

As in Paper I, we extrapolated the CME trajectories at $1.8 R_{\odot}$ to show how these transients correlated in time with the associated type IIs. Results are plotted in Fig. 4a, which shows the ratio between type II and CME speeds versus delay times between CMEs and type IIs at $1.8 R_{\odot}$ with CME trajectories fitted with and without data from other instruments in the inner corona. Out of the ten CME/type II events considered in this study, only one type II burst appeared to precede the CME leading edge, even after the problem of the bias introduced by the extrapolation method was removed by integrating the LASCO data with the new set of complementary observations at low coronal heights. As for the remaining events, the type II sources were lagging well behind the leading edge of the CMEs, although in general we notice that the time lags obtained with the help of the new set of observations appear to be diminished sensibly in at least a few cases. This means that acceleration effects can be significant at the typical coronal shock heights and must be taken into account for a proper analysis of the CME/shock relationship. Notwithstanding the above, this analysis confirms that the leading edges actually appear to precede the shocks, independent of the possibility that the extrapolation method adopted in Paper I overestimated (or underestimated) the actual CME trajectories at the typical type II heights.

As for the problem of the speed correlation, Fig. $4 \mathrm{~b}$ shows the type II versus CME speeds at $1.8 R_{\odot}$ for all ten events with CME trajectories fitted with and without data from other instruments at lower heights. In both cases the two speeds remain uncorrelated (reasonably, we would expect the shock-generated type II emission speeds to be at least equal or higher, within the errors, than the associated CME speeds). Figure $4 \mathrm{c}$ shows a similar correlation plot but one obtained applying the CME-driven shock front-flank model presented in Paper I. In this model, the shock surface propagates hemispherically just above a conically expanding bubble-type CME leading edge and the shock strength is enhanced (thus favoring type II emission) along a 

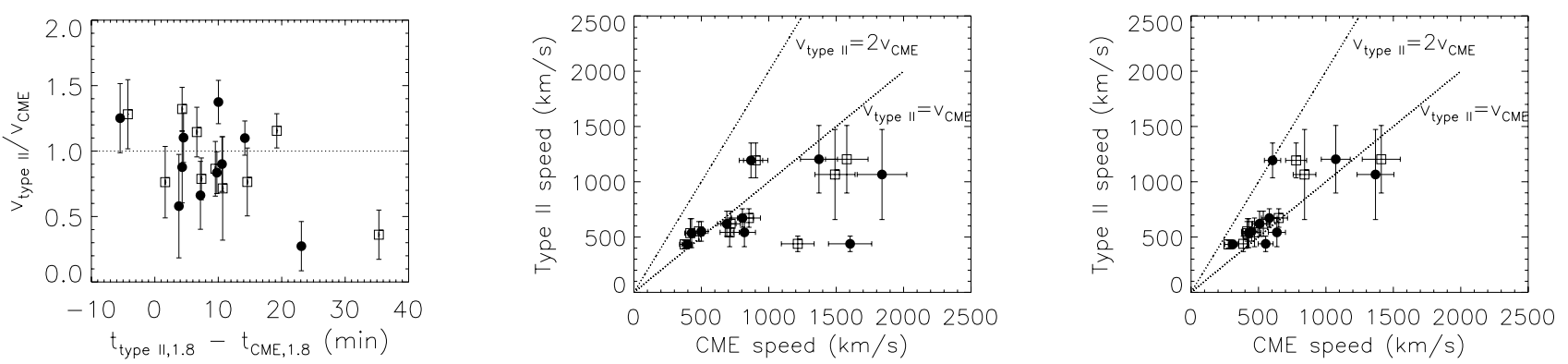

Fig. 4. a) Ratio between type II and CME speed versus delay times between CMEs and type IIs at $1.8 R_{\odot}$ for the events listed in Table 1 with CME trajectories fitted without (squares) and with (filled circles) data from other instruments at lower heights. b) Plot of the type II speeds versus CME speeds at $1.8 R_{\odot}$ for the same events with CME trajectories fitted without (squares) and with (filled circles) data from other instruments at lower heights. c) Same as for Fig. 4b, but adopting the angle correction that minimizes the time lag between the type II and CME leading edge at a (projected) height of $1.8 R_{\odot}$ along the streamer's axis direction.

direction (corresponding to the low- $v_{A}$ streamer axis) that forms an angle different from zero with respect to the axis of symmetry of the expanding CME. After finding the angle corrections that minimize the time lags between the type II and the CME leading edges at a (projected) height of $1.8 R_{\odot}$ along the streamer's axes directions for all events, we still find a good correlation between the two speeds, as required in the CME-driven shock scenario.

The result of this analysis, in the picture of the CME-driven origin interpretation of coronal shocks, is that unconsidered accelerations in the lower corona corresponding to typical type II heights did not affect in an important way the results obtained in Paper I and that the reason for the observed mismatch between the CME and the shock dynamics must be sought elsewhere, most probably, as stated by Mancuso \& Raymond (2004), in effects of geometry.

\section{Conclusions}

In the companion Paper I, Mancuso \& Raymond (2004) analyzed a sample of $\mathrm{CME} /$ shock events to show that the computed type II burst locations were consistent with CME-driven shocks. The apparent lack of relationship, typically found between speeds, times, or extrapolated spatial positions for metric type II bursts and CMEs was attributed to geometrical effects, favoring emission at the flanks of the shock surfaces in correspondence with denser low-Alfvén-speed coronal structures, where shock strengths are enhanced. The conclusions that supported the CME-driven shock front-flank scenario were based, however, on the sunward extrapolation of the CME trajectories deduced from analyzing LASCO observations in the outer corona that cannot be accurate at low coronal heights due to significant accelerations.

In the present paper, we re-examined the above relationship by taking into account complementary CME observations of a subset of ten events from the original sample obtained by other instruments (EIT, UVCS, and Mk4) in the inner corona, in order to investigate whether previously undetected accelerations at low coronal heights below the LASCO C2 coronagraph field of view (and not effects of geometry, as proposed to Paper I) could have caused the observed mismatch between the CME/type II dynamics. By using the full set of observations in the inner and outer corona, we still found that the CME trajectories appear to precede the shocks, independent of the fact that the adopted extrapolation method applied to the LASCO data might have overestimated (or underestimated) the actual CME heights. Also, the correlation between the type II and the CME speeds, obtained by fitting all data points with a quadratic polynomial, did not improve, although acceleration effects are found to be important at the typical metric type II heights and must be taken into account for a proper analysis of the CME/shock relationship. If we accept the CME-driven shock scenario for metric type II bursts, we did rule out the possibility that unaccounted accelerations below the LASCO C2 occulter could be the sole responsible for the observed mismatch between the CME and the shock dynamics. The reason for the observed discrepancy must be sought elsewhere, most probably, as stated in Paper I, in effects of geometry.

Acknowledgements. We thank the anonymous referee for comments that helped to improve the manuscript. The CME catalog is generated and maintained by the Center for Solar Physics and Space Weather, The Catholic University of America in cooperation with the Naval Research Laboratory, and NASA. SOHO is a project of international cooperation between ESA and NASA. The Mauna Loa Solar Observatory (MLSO) is operated by the High Altitude Observatory (HAO), a division of the National Center for Atmospheric Research (NCAR), which is sponsored by the National Science Foundation (NSF).

\section{References}

Bemporad, A., Poletto, G., Suess, S. T., et al. 2003, ApJ, 593, 1146 Brueckner, G. E., Howard, R. A., Koomen, M. J., et al. 1995, Sol. Phys., 162, 357

Delaboudinière, J. P., Artzner, G. E., Brunaud, J., et al. 1995, Sol. Phys., 162, 291

Elmore, D. F., et al. 2003, Proc. SPIE, 4823

Gallagher, P. T., Lawrence, G. R., \& Dennis, B. R. 2003, ApJ, 588, L53

Kahler, S. W., \& Reames, D. V. 2003, ApJ, 584, 1063

Kohl, J. L., Esser, R., Gardner, L. D., et al. 1995, Sol. Phys., 162, 313

Mancuso, S., \& Raymond, J. C. 2004, A\&A, 413, 363

Mancuso, S., Raymond, J. C., Kohl, J., et al. 2002, A\&A, 383, 267

Mancuso, S., Raymond, J. C., Kohl, J., et al. 2003, A\&A, 400, 347

Neupert, W. M., et al. 2001, J. Geophys. Res., 106, 25215

Newkirk, G. A. 1961, ApJ, 133, 983

Saito, K. 1970, Ann. Tokyo Astron. Obs., 12, 53

Shanmugaraju, A., Moon, Y.-J., Dryer, M., \& Umapathy, S. 2003, Sol. Phys., 215,185

Sheeley, N. R., Howard, R. A., Michels, D. J., et al. 1985, J. Geophys. Res., 90, 163

St. Cyr, O. C., et al. 1999, J. Geophys. Res., 104, 12493

Uchida, Y. 1974, Sol. Phys., 39, 431

Wagner, W. J., \& MacQueen, R. M. 1983, A\&A, 120, 136

Yashiro, S., et al. 2002, AGU Spring Meeting

Zhang, J., Dere, K. P., Howard, R. A., et al. 2001, ApJ, 559, 452 